\title{
TARJETAS DE RESPUESTA ¿Son necesarias para una correcta cumplimentación del cuestionario?
}

\section{RESPONSE CARDS}

Are they necessary for correctly filling in a questionnaire?

\author{
VIDAL DíAZ de RADA IgúZquIZA vida@unavarra.es \\ Universidad Pública de Navarra. España
}

\begin{abstract}
Resumen
La utilización en encuestas de preguntas con tarjetas de respuesta está totalmente aceptada por la comunidad investigadora. Esto supone una carga de trabajo "extra" en la tarea del entrevistador, lo que explica que en ocasiones no se utilicen correctamente. Pese a esta situación, hay muy poca literatura sobre la influencia de las tarjetas en las respuestas del entrevistado. El objetivo de este trabajo es profundizar en los efectos que la utilización de tarjetas tiene en la calidad de las respuestas del cuestionario, partiendo de la hipótesis que considera que las tarjetas - pese a complicar la tarea del encuestador-suponen importantes mejoras en la administración del cuestionario. Utilizaremos para ello un estudio del Centro de Investigaciones Sociológicas con 23 preguntas de tarjeta, comparando las respuestas de los entrevistados que utilizaron las tarjetas con aquellos que no las emplearon.
\end{abstract}

\section{Palabras Clave}

Calidad de la respuesta; Encuesta presencial; Efectos producidos por el orden de las respuestas; Entrevistadores; No Respuesta parcial; Preguntas de tarjeta.

\section{Abstract}

Using "response cards" in question surveys is unanimously approved by the research community. The fact that this represents an extra workload for the interviewer's task explains why they sometimes are not used correctly. Despite this situation there is a paucity of literature on the influence of the response card on the respondent's answers. The aim of this study is to deepen the analysis of how using these cards affect the quality of the survey's responses. To do so, we start from the assumption that the cards -while complicating the interviewer's task, result in significant improvements in the survey's administration. For this purpose we will use a study with 23 card questions (question cards) by the Centro de Investigaciones Sociológicas, (the Spanish Centre for Sociological Research), and we will compare the answers of respondents that used cards with those who did not.

\section{KEYWORDS}

Face to Face Survey; Interviewer; Item Non Response; Response Card Question; Response Order Effects Survey Quality. 


\section{INTRODUCCIÓN}

En la actualidad nadie duda de la adecuación de las "tarjetas de respuesta" en la investigación con encuesta y, de hecho, una de las mayores debilidades de otras modalidades como la encuesta telefónica es, precisamente, la imposibilidad de mostrar esta información al entrevistado (Groves y Kahn 1979). El empleo de "preguntas con tarjeta" se fundamenta en la idea de que presentar una información visual con las categorías de respuesta estimula el recuerdo del entrevistado, permitiendo obtener respuestas más precisas. Esta forma de proceder está recomendada cuando la pregunta presenta un gran número de opciones, largas respuestas, o cuando el entrevistado debe ordenar las respuestas (para lo que precisa considerar conjuntamente todas ellas).

Pese a lo acertado de este planteamiento se ha investigado muy poco sobre los efectos que tiene su utilización en las respuestas de los entrevistados. Se desconoce si el empleo de las tarjetas supone una mejora en la respuesta del cuestionario -al disminuir la no respuesta parcial -0 si, por otro lado, estas "producen" determinadas respuestas que implican un descenso en la calidad de respuesta al no recoger lo que realmente piensa/hace/considera el entrevistado. Este es el objetivo del presente trabajo: profundizar en los efectos que la utilización de tarjetas tiene en la calidad de las respuestas asumiendo como hipótesis de partida la de que las tarjetas - pese a complicar la tarea del encuestador (ya de por sí muy difícil)— aportan importantes mejoras en el proceso de respuesta del cuestionario. Utilizaremos, para ello, un estudio del Centro de Investigaciones Sociológicas (en adelante, CIS) comparando las respuestas de los entrevistados que utilizaron "tarjetas" con las de aquellos que no las emplearon. Se trata, concretamente, del Barómetro de marzo del año 2011 (Estudio 2864) que trata sobre la prestación de ayuda voluntaria y que tiene 23 preguntas de tarjeta, aunque en realidad son 20 tarjetas, puesto que tres comparten el mismo texto.

\section{EMPLEO DE INFORMACIÓN ORAL Y VISUAL EN LA RESPUESTA DE CUESTIONARIOS}

El proceso de comunicación entre entrevistador y encuestado puede hacerse de forma "oral", al "leer" el entrevistador las preguntas, y respuestas del cuestionario, o "visual", fruto de la "lectura" del entrevistado de la información presentada en las tarjetas. Utilizar una u otra puede generar notables cambios en la respuesta (Dillman et al. 2009), aunque

\footnotetext{
${ }^{\star}$ El autor agradece las sustanciales mejoras aportadas por el Consejo Editorial y por dos evaluadores anónimos. Gracias también al Banco de Datos del CIS por proporcionar información (anonimizada) sobre el comportamiento de los entrevistadores. Este texto investigación es parte de una investigación financiada por el Ministerio de Economía y Competitividad, ref. CSO2012-34257.
} 
este trabajo limita su objeto de estudio a la no respuesta parcial, planteando como hipótesis que la no utilización de recursos visuales produce un aumento del número de preguntas no respondidas.

La investigación sobre "no respuesta parcial" (preguntas sin responder) es un ámbito de estudio con gran tradición en encuestas sobre actitudes, preguntas predominantes en la investigación social. Desde que Converse (1964) descubriera que las respuestas de algunos entrevistados no desvelan "actitudes reales", numerosos científicos han abordado el estudio de este fenómeno. Preguntar sobre aspectos sobre los que nunca se ha reflexionado debería generar respuestas "no sabe" o "no tiene opinión", pero la escasa presencia de respuestas de este tipo en varias investigaciones realizadas por Converse le llevó a plantear que un gran número de entrevistados consideran que estas respuestas desvelan ignorancia, lo que genera incomodidad. Así, en vez de responder "no sabe", los que se encuentran en esta situación seleccionan aleatoriamente, una de las categorías de respuesta ofrecidas. Esta autora lo explica aludiendo a que nadie desea ser catalogado como ignorante y, por ello, resulta más sencillo seleccionar una de las opciones ofrecidas por el entrevistador.

La gran relevancia de este descubrimiento ha llevado a numerosos investigadores a recomendar la colocación de una categoría "no sabe" para los que desconocen la respuesta (entre otros, Berdie y Anderson 1974; Converse y Presser 1986; Oppenheim 1992), lo que puede generar también que esta sea seleccionada por entrevistados - con opiniones formadas - que no desean pensar sobre el tema (Survey Research Laboratory 1994; Schuman y Presser 1996). De hecho numerosos estudios han demostrado que una gran parte de los entrevistados que dicen "no sabe" cuando se ofrece esta opción, proporcionan información a preguntas de la misma temática cuando esta respuesta no está presente (Bishop et al. 1983; Gilljam y Granberg 1993; Schuman y Presser 1996). Las investigaciones realizadas por Gilljam y Granberg (1993) demuestran que, cuando se presenta la opción "no sabe" o "no contesta", entre el $20 \%$ y el $30 \%$ de las respuestas conseguidas por esta categoría son de personas que tienen una actitud pero que no desean darla a conocer; aunque en otras investigaciones han aumentado esta cifra hasta el $43 \%$ (Gilljam y Granberg 1993).

Esta situación explica que los cuestionarios del CIS no presenten esta categoría a los entrevistados, aunque esté disponible en el cuestionario para que el entrevistador la anote cuando sea elegida. De hecho, el manual del entrevistador de este organismo indica de forma específica que "[...] (los entrevistadores) deben formular literalmente las preguntas y anotar las respuestas, no ofreciendo las opciones "no sabe" o "no responde", pero anotándolas cuando son elegidas" (Centro de Investigaciones Sociológicas 2011). En este trabajo se diferenciará entre los dos tipos de no respuesta: no saber la respuesta ("no sabe") y la manifestación clara de no responder ("no responde"). 


\section{Aplicación a un CAso. El Barómetro de marzo del año 2011 (EsTUDIO CIS NÚMERO 2864)}

\section{Fuente de datos}

El Estudio 2864 sigue el formato habitual de los barómetros del CIS. Las 2461 entrevistas realizadas en marzo del año 2011 —de las 2500 planificadas — implican un error muestral del $\pm 2,0 \%$ para el conjunto de la muestra y en el supuesto de muestreo aleatorio simple (nivel de confianza del $95,5 \%$ y $P=Q$ ). ${ }^{1}$

El cuestionario consta de 44 preguntas (118 variables) y los entrevistados emplearon un promedio de 18 minutos en responderlo². Comienza, como es habitual en los barómetros del CIS, con seis preguntas sobre la valoración de la situación económica y política (actual y retrospectiva a un año), principales problemas del país, y problemas que más afectan al entrevistado. A continuación se procede con el tema específico del barómetro de marzo, el trabajo voluntario, al que se dedican 33 preguntas que proporcionan 85 respuestas/variables ${ }^{3}$. Se trata de un cuestionario que emplea una gran variedad de tipos de preguntas (Azofra 1999) tanto considerando la libertad de la respuesta (abierta, cerrada y semicerrada), número de respuestas (única y múltiple, y dentro de esta si es dicotómica o categórica), tipo de respuesta (dicotómica, categórica, respuesta ordinal, escala numérica y valoración), "directividad" de la pregunta (directas e indirectas) y tipos de preguntas según su función en el cuestionario: baterías, preguntas filtro, tarjetas, etc. (Díaz de Rada 2001).

Tras las preguntas sobre trabajo voluntario el cuestionario prosigue con un conjunto de preguntas que se repiten todos los meses: ideología, voto a las elecciones generales del 9 de marzo, variables sociodemográficas (sexo, edad, nivel de estudios, estado civil, relación con la actividad y ocupación), creencia religiosa y nivel de práctica, evaluación de la situación económica actual, persona que aporta más ingresos al hogar, probabilidad de perder/encontrar empleo en el próximo año, y dominio del castellano (14 preguntas, 21 variables).

Tres argumentos nos llevaron a la elección de este estudio. Tras analizar las características de los entrevistados que utilizan y no utilizan las tarjetas de respuesta en todos los barómetros del año 2011, este era el que presentaba mues-

\footnotetext{
${ }^{1}$ Los detalles técnicos de la investigación se muestran en: http://www.cis.es/cis/export/sites/default/Archivos/Marginales/2860_2879/2864/Ft2864.pdf

${ }^{2}$ Ningún entrevistado ha respondido todo el cuestionario porque había preguntas específicas para determinados subgrupos. Así, los entrevistados que nunca habían realizado trabajo voluntario respondieron las preguntas 12-15, "saltando" de ahí a la pregunta 20 , mientras que los que habían sido voluntarios en los últimos doce meses pasaron de la pregunta 11 a la 16.

${ }^{3}$ En el Anexo 1 se detallan todos los temas tratados en el estudio.
} 
tras más similares, más equivalentes (Petersen 2008). No menos importante es la variabilidad en el tipo de preguntas empleadas, la presencia de diferentes tipos de preguntas, tal y como se ha señalado dos párrafos más atrás. El tercer argumento, sin duda el más importante considerando el tema central del artículo, es la gran presencia de preguntas con tarjetas, así como la variabilidad en el tipo de tarjetas empleadas.

\section{Variables objeto de esta investigación}

El estudio de la influencia de las tarjetas precisa, en primer lugar, catalogar las preguntas del cuestionario según el tipo de tarjetas empleado. Para ello se clasificarán las tarjetas según tres criterios (cuadro 1). El primero considera conjuntamente el número de categorías de respuesta y la "cantidad" de información incluida en la tarjeta, distinguiendo cuatro grupos: tres categorías de respuesta (y menos de 10 palabras), entre cuatro y seis categorías de respuesta (entre 22 y 40 palabras), entre ocho y once categorías de respuesta y alrededor de 50 palabras (entre 47 y 66), y 12 categorías de respuesta y alrededor de 100 palabras. Esta clasificación, que ha mostrado su eficacia en investigaciones realizadas en otros contextos (Holbrook et al. 2007), ordena las preguntas en función de su facilidad para responder: pocas opciones de respuesta en el primer grupo (que incluso hace dudar de la necesidad "real" de la tarjeta) y un elevado número de opciones en el cuarto grupo que hace "imprescindible" la utilización de una tarjeta para que el entrevistado recuerde todas las respuestas. En el cuadro 1 se muestran las preguntas clasificadas en cada grupo, el tipo de tarjeta empleada, número de opciones de respuesta y cantidad de información transmitida (palabras y caracteres).

El segundo criterio, denominado "largas respuestas en dos categorías", considera una pregunta con dos largas opciones de respuesta, dos frases que son presentadas a los entrevistados para que indiquen su acuerdo con una u otra. La pregunta presenta únicamente dos categorías de respuesta, y no ha sido incluida en el primer grupo del primer criterio ("tres categorías", ver cuadro 1) por el tamaño de la respuesta, por la enorme cantidad de información transmitida. De hecho, se trata de un tamaño que se asemeja más al segundo grupo del primer criterio. Se ha optado por clasificarlo por separado porque, aun cuando el número de palabras es similar, resulta mucho más sencillo elegir entre un par de categorías que entre las seis opciones que ofrecen, por ejemplo, las preguntas 22 y 32 (clasificadas en el segundo grupo).

El tercer criterio se refiere a tres preguntas cuya tarjeta es una escala "visual", una "regla" en la cual los entrevistados se posicionan considerando la dimensión izquierda y derecha; y que - se plantea como hipótesis - la observación o no de la tarjeta puede implicar la elección de respuestas más extremas, más centradas 0 más fáciles. 


\section{Cuadro 1.}

Clasificación de las preguntas del cuestionario según el tipo de tarjetas empleado.

Primer criterio: Número de categorías de respuesta y número de palabras

Preg. $7\left(^{*}\right)$ : frecuencia realización diversas actividades sin ánimo de lucro (tarjeta A; 3 categorías: últimos 12 meses, con anterioridad, nunca)

CUATRO, CINCO Y SEIS CATEGORÍAS (entre 22 y 40 palabras, 100-268 caracteres)

Preg. 10: frecuencia de realización de trabajo voluntario en el último año (tarjeta C, 4 categorías cortas, 22 palabras, 100 caracteres)

Preg. 16: frecuencia de realización de trabajo voluntario en el último año durante su etapa de más implicación (tarjeta C, 4 categorías cortas, 22 palabras, 100 caracteres)

Preg. 22: motivos por el que hay personas que NO realizan labores de voluntariado (tarjeta $\mathrm{H}, 6$ categorias medianas, 40 palabras, 268 caracteres)

Preg. 32: recuerdo de votar o abstenerse en las últimas elecciones generales (tarjeta Recuerdo de voto, 5 categorias medianas, 27 palabras, 120 caracteres)

ENTRE OCHO Y ONCE CATEGORÍAS (entre 47 y 66 palabras, 319-375 caracteres)

Preg. 11: razón por la que dejó de hacer trabajo voluntario (tarjeta D, 9 categorías largas, 58 palabras, 355 caracteres)

Preg. 12: razones por las que nunca ha realizado trabajo voluntario (tarjeta E, 10 categorías largas, 66 palabras, 375 caracteres)

Preg.18: motivos por los que usted realizaba labores de voluntariado (tarjeta G, 11 categorias largas, 47 palabras, 319 caracteres)

Preg. 21: motivos por el que hay personas que realizan labores de voluntariado (tarjeta G, 11 categorias largas, 47 palabras, 319 caracteres)

Preg. 28: acuerdo con la necesidad de la reforma del sistema de pensiones (tarjeta 0, 3 categorias largas, 52 palabras, 304 caracteres)

DOCE CATEGORÍAS (alrededor de 100 palabras)

Preg. 8: realización de labores de voluntariado en diversas áreas (tarjeta B, 12 categorías largas, 97 palabras, 761 caracteres)

Preg. 15: áreas en las que prestaría trabajo voluntario (tarjeta B, 12 categorías largas, 97 palabras, 761 caracteres)

Segundo criterio: largas respuestas en dos categorías

Preg. 23: tarjetas I, J, K, I, M con dos frases cada una:

a) Valoración del papel de la formación en la labor del voluntariado (31 palabras, 200 caracteres);

b) Papel del voluntariado en relación a la cobertura de los servicios públicos (32 palabras, 208 caracteres);

c) Labor resolutiva del voluntariado (22 palabras, 155 caracteres);

d) Papel de la organización en la labor del voluntariado (39 palabras, 252 caracteres) y

e) Reconocimiento social de la labor del voluntariado (19 palabras, 134 caracteres)

\section{Tercer criterio: tarjeta con escala visual}

Preg. 26: Escala de valoración de la reforma del sistema de pensiones (tarjeta $\mathrm{N}$, escala $0-10$, con dos palabras a cada lado)

Preg. 30: Escala de confianza en la gente (tarjeta P, escala 0-10, con dos frases a cada lado)

Preg. 31: Escala ideología (tarjeta Escala, escala 1-10, con una palabra a cada lado)

${ }^{*}$ ) Puede consultarse el texto completo de cada pregunta, así como el contenido de las tarjetas de respuesta, en la siguiente dirección web: http://www.cis.es/cis/export/sites/default/-Archivos/Marginales/2860_2879/2864/cues2864.pdf Fuente: Centro de Investigaciones Sociológicas 2011, Estudio n. ${ }^{\circ} 2864$. 


\section{Delimitación de objetivos y técnicas de análisis utilizadas}

El objetivo principal del trabajo es conocer los efectos de la utilización de tarjetas en la calidad de las respuestas de un cuestionario, centrando la atención en la respuesta parcial, en el número de preguntas no respondidas. Para ello se considerará la media de no respuestas proporcionadas por los entrevistados que han respondido con y sin tarjetas en cada grupo de preguntas del cuadro 1, diferenciando "no sabe" y "no responde". Para conocer si existen diferencias entre ambos grupos se utilizará un contraste de hipótesis considerando la significación de la diferencia de medias de ambas poblaciones, tal y como han procedido investigaciones llevadas a cabo en otros contextos (entre otros, Bishop y Smith 2001; Dillman et al. 2001; Krosnick et al. 2002; Holbrook, Green y Krosnick 2003; Holbrook et al. 2007; Heerwegh 2009; Heerwegh y Loosveldt 2008; Chang y Krosnick 2010). La sensibilidad de este contraste a la diferencia de varianzas recomienda precisar, en cada contraste, si la comparación se ha realizado en condiciones de homocedasticidad o heterocedasticidad; utilizando el test de Levene para identificar cada situación.

Con el fin de detectar hasta qué punto estas diferencias pueden estar producidas no tanto por el tipo de tarjeta sino por la desigual composición sociodemográfica de las muestras se empleará la técnica de la regresión con respuesta binaria, al considerar como término dependiente la presencia/ausencia de "no respuesta parcial". Los términos independientes de la regresión serán el sexo, edad, nivel de estudios, relación con la actividad, y utilización o no de tarjetas. Las variables cualitativas han sido codificadas considerando la última categoría como referencia (véanse las tablas 3, 6 y 9), y sus coeficientes se interpretan como el efecto que produce en la variable dependiente un cambio de una unidad en cada variable independiente manteniendo constantes todas las demás.

Terminada la exposición sobre las técnicas de análisis, una breve explicación sobre cómo se ha operado para diferenciar los que han respondido con y sin tarjetas. Para conocer la influencia de las tarjetas es preciso contar con dos muestras: una que haya respondido utilizándolas y otra que no las haya empleado. La pregunta 50 del cuestionario plantea a los encuestadores que señalen si el entrevistado ha utilizado todas las tarjetas, solo algunas, o si las tarjetas han sido leídas por el entrevistador ${ }^{4}$. El 53,7 \% de los entrevistados (1321 personas) ha utilizado todas, el $23,7 \%$ algunas (583) y el $22 \%$ (539 entrevistados) no ha utilizado ninguna y ha sido el encuestador el que ha leído la información de las tarjetas. De modo que el primer grupo ha respondido las preguntas en base a la información "visual" mostrada en las tarjetas, mientras que el tercero ha recibido únicamente estímulos "orales", estímulos que — planteamos como hipótesis_

\footnotetext{
${ }^{4}$ Pregunta 50: Respecto a las tarjetas...

- La persona entrevistada las ha usado todas.

- Solo ha usado algunas.

Las he leído yo.
} 
pueden no ser muy eficaces cuando se transmiten largas informaciones como las mostradas en algunas tarjetas, fundamentalmente las que tienen más de ocho opciones de respuesta y aquellas que incluyen muchas palabras. Respecto al segundo grupo, que utiliza algunas tarjetas, al no poder precisar cuáles ha utilizado y cuáles no, decidimos eliminarlo de los análisis.

De modo que las 2461 entrevistas realizadas en el Barómetro de marzo del 2011 se reducen a 1860 en la presente investigación. Un análisis detallado de la distribución de las variables sociodemográficas desvela diferencias entre los grupos, por lo que se procedió a ponderar la muestra de los que utilizan tarjetas a fin de trabajar con dos muestras equivalentes en relación al sexo, edad, nivel de estudios y relación con la actividad. Las cuatro variables fueron utilizadas para la ponderación; reduciendo el tamaño del primer grupo hasta 542 entrevistados 5 . Finalizamos este apartado metodológico señalando que la exposición de resultados se realizará siguiendo la clasificación presentada en el cuadro 1 , lo que permitirá conocer el efecto de cada tipo de tarjeta.

\section{INFLUENCIA DE LAS TARJETAS CONSIDERANDO LA CANTIDAD DE CATEGORÍAS Y NÚMERO DE PALABRAS}

El primer criterio de clasificación de las preguntas -y sus correspondientes tarjetas- tiene en cuenta la "cantidad" de información considerando el número de categorías de respuesta y el número de palabras dentro de cada una. Con este criterio se distinguieron cuatro grupos: tres categorías de respuesta (menos de 10 palabras); cuatro, cinco y seis categorías de respuesta (entre 22 y 40 palabras); entre ocho y once categorías de respuesta y alrededor de 50 palabras (entre 47 y 66); y doce categorías de respuesta y alrededor de 100 palabras.

\section{3.a. Tarjetas con menor número de categorías de respuesta}

En la tabla 1 se muestra el número "medio" de no respuestas de las preguntas con tres categorías de respuesta, el número de no respuestas dividido entre el número de entrevistados ${ }^{6}$. En este caso la tabla está referida a una única pregunta, la pregunta 7 , que consulta a los entrevistados por la frecuencia de realización de seis actividades sin ánimo de lucro, pudiendo responder "últimos 12 meses", "con anterioridad" y "nunca".

Como puede apreciarse el número medio de no respuestas es extremadamente reducido, aunque es menor entre los que usan tarjetas que entre los que las escuchan

${ }^{5}$ La distribución de la muestra se presenta en el Anexo 2.

${ }^{6}$ Así, por ejemplo, el valor 0,0093 de "no responde" (Tabla 1) en los entrevistados que no utilizan tarjetas es la ratio del número de respuestas "no responde" (5) entre el número de entrevistados (539). La información sobre el número de entrevistados que no utilizan las tarjetas está en el penúltimo párrafo del apartado "Delimitación de objetivos y técnicas de análisis utilizadas". 
(diferencia 0,006 no significativa). El número de respuestas "no sabe" es ligeramente superior al "no responde". Cuando se comparan ambos grupos se desvela que, aunque no hay diferencia significativa, los que "escuchan" el texto de las tarjetas proporcionan más respuestas "no sabe" $(0,0033)$.

El número de preguntas sin respuesta, aun siendo un indicador importante de calidad en la cumplimentación del cuestionario, está determinado -lógicamentepor el número de preguntas planteadas. Con el fin de ofrecer una medida más estable -y de más fácil interpretación- se calculará el porcentaje de no respuestas considerando todos los ítems de la pregunta. Es decir, no se trata únicamente de conocer la media de ítems sin responder sino, más bien, el porcentaje de no respuestas en una pregunta formada por varios ítems y que ha sido respondida utilizando un determinado tipo de tarjeta. Se explicará su cálculo tomando como ejemplo la pregunta de la tabla 1. La primera parte de la tabla recoge el número de no respuestas detectadas en preguntas con tres categorías de respuesta, que en

Tabla 1.

Media de no respuestas de las preguntas con tres categorias de respuesta

(menos de 10 palabras, 34 caracteres)

\begin{tabular}{|c|c|c|c|c|c|}
\hline \multirow[t]{2}{*}{ Pregunta utilizada: } & \multicolumn{5}{|c|}{$\begin{array}{l}\text { Preg. } 7 \text {, frecuencia realización diversas actividades sin ánimo de lucro (tarjeta A; } 3 \text { catego- } \\
\text { rías: últimos } 12 \text { meses, con anterioridad, nunca). }\end{array}$} \\
\hline & $\begin{array}{c}\text { Usa } \\
\text { tarjetas } \\
\text { (lee tarjeta) }\end{array}$ & $\begin{array}{c}\text { Tarjetas } \\
\text { leídas } \\
\text { (escucha } \\
\text { información } \\
\text { de la tarjeta) }\end{array}$ & t-valor & $p$ & $\begin{array}{l}\text { Igualdad } \\
\text { varianzas } \\
\text { (Levene) }\end{array}$ \\
\hline \multicolumn{6}{|l|}{ No responde } \\
\hline Número & 0,0030 & $0,0093\left(^{*}\right)$ & 1,237 & 0,216 & No \\
\hline Porcentaje $\left(^{*}\right)$ & $0,0497 \%$ & $\left.0,1546 \%{ }^{(* *}\right)$ & & & \\
\hline \multicolumn{6}{|l|}{ No sabe } \\
\hline Número & 0,0153 & 0,0186 & 0,402 & 0,688 & Sí \\
\hline Porcentaje ${ }^{* \star}$ ) & $0,2551 \%$ & $0,3092 \%$ & & & \\
\hline
\end{tabular}

Notas:

$\left({ }^{*}\right)$ La media de "no respuesta" es el número absoluto de no respuestas respecto al número de entrevistados que no emplea tarjetas $(5 / 539)$.

$\left.{ }^{*}\right)$ La tasa de "no respuesta" es el número medio de no respuestas respecto al total de respuestas de esta pregunta, multiplicado por 100. En este caso 5 entre los 3234 (539 entrevistados por los seis ítems) que han respondido esta pregunta.

Fuente: Elaboración propia con datos del CIS 2011, Estudio n. ${ }^{0} 2864$. 
este caso corresponde a una única pregunta; la pregunta 7. Los $539^{7}$ entrevistados que NO usan tarjetas han generado cinco "no respuestas" en toda la pregunta 0 , lo que es lo mismo, una media de 0,0093 de no respuestas (5/539). Obsérvese que se trata de la media conjunta considerando las seis actividades preguntadas porque se ha utilizado la misma tarjeta para todas, y no la media de cada actividad (ítem) que varía enormemente de unas a otras.

La distribución porcentual de respuestas se calculará dividiendo el número de no respuestas ( 5 entre los que NO usan tarjetas) entre las 3234 respuestas que deben generar esta pregunta (539 por seis ítems) y multiplicado por 100 . Así en la primera parte de la tabla 1 puede apreciarse que los que no usan tarjetas dejan sin responder el $0,1546 \%$ de las preguntas, porcentaje que triplica las no respuestas de los que usan tarjetas $(0,0497 \%)$. En cualquier caso, la agregación de ambos indicadores proporciona una no respuesta inferior al $0,05 \%$ de las respuestas ${ }^{8}$ de la pregunta, existiendo ligeras diferencias (nunca significativas) entre los que responden utilizando y sin utilizar las tarjetas.

\section{Tarjetas con entre cuatro y seis categorías de respuesta}

El segundo grupo de preguntas incluye aquellas que tienen cuatro, cinco y seis categorías de respuesta con menos de 50 palabras, que consideradas conjuntamente presentan un rango de entre 22 y 40 palabras. A este grupo pertenecen la pregunta 10 y la 16 , ambas con cuatro categorías de 22 palabras ( 100 caracteres), la pregunta 22 con 6 categorías y 40 palabras (268 caracteres), y la pregunta 32 con 27 palabras ( 120 caracteres). Comenzando con la no respuesta parcial, las 0,1444 no respuestas proporcionadas por los entrevistados con tarjetas supone un 3,6\% de las respuestas, que aumenta al 4,73 $\%$ entre los que no las usaron (tabla 2), aunque la diferencia no llega a ser significativa. Esta diferencia, sin embargo, es importante en el número de respuestas "no sabe", al aumentar del 0,0445 entre los que usan tarjetas a 0,1058 entre los que no las usan; diferencia significativa al $99 \%$.

En el apartado "Delimitación de objetivos y técnicas de análisis utilizadas" se explicó que la regresión múltiple sería utilizada con el fin de delimitar hasta qué punto las diferencias entre ambos colectivos es producida por la utilización de tarjetas 0 por la diferente composición sociodemográfica de las muestras. Tal y como se señaló anteriormente se utilizará la regresión logística debido al escaso número de no respuestas $^{9}$, utilizando el valor 1 cuando el entrevistado responde todas las preguntas

\footnotetext{
${ }^{7}$ Número de entrevistados que no utilizan tarjetas, tal y como se ha señalado en el penúltimo párrafo del apartado "Delimitación de objetivos y técnicas de análisis utilizadas".

${ }^{8}$ Concretamente del $0,3048 \%(0,0497+0,2551)$ en el caso de los que usan tarjetas y del $0,4638 \%$ entre los que no las utilizan.

${ }^{9}$ En el caso concreto de estas preguntas la mayor parte de los entrevistados (2074 de los 2461 entrevis-
} 
Tabla 2.

Media de no respuestas de las preguntas con cuatro, cinco y seis categorías de respuesta (entre 22 y 40 palabras, 100-268 caracteres)

Preguntas utilizadas:

Preg. 10: frecuencia de realización de trabajo voluntario en el último año (tarjeta C, 4 categorías cortas, 22 palabras, 100 caracteres).

Preg. 16: frecuencia de realización de trabajo voluntario en el último año durante su etapa de más implicación (tarjeta C, 4 categorías cortas, 22 palabras, 100 caracteres).

Preg. 22: motivos por el que hay personas que NO realizan labores de voluntariado (tarjeta $\mathrm{H}, 6$ categorías medianas, 40 palabras, 268 caracteres).

Preg. 32: recuerdo de votar o abstenerse en las últimas elecciones generales (tarjeta Recuerdo de voto, 5 categorías medianas, 27 palabras, 120 caracteres).

\begin{tabular}{|c|c|c|c|c|c|}
\hline & $\begin{array}{c}\text { Usa } \\
\text { tarjetas }\end{array}$ & $\begin{array}{l}\text { Tarjetas } \\
\text { leídas }\end{array}$ & t-valor & $p$ & $\begin{array}{c}\text { Igualdad } \\
\text { varianzas } \\
\text { (Levene) }\end{array}$ \\
\hline \multicolumn{6}{|l|}{ No responde } \\
\hline Número & 0,1444 & 0,1892 & 1,917 & 0,055 & No \\
\hline Porcentaje & $3,6102 \%$ & $4,7310 \%$ & & & \\
\hline \multicolumn{6}{|l|}{ No sabe } \\
\hline Número & 0,0445 & 0,1058 & 3,790 & 0,000 & No \\
\hline Porcentaje & $1,1125 \%$ & $2,6438 \%$ & & & \\
\hline
\end{tabular}

Fuente: Elaboración propia con datos del CIS 2011, Estudio n. ${ }^{0} 2864$.

y codificando la presencia de respuesta parcial con el 0 . No se diferenciará entre los entrevistados que dejan sin responder (o responden no sabe) uno o varios items por el escaso número de personas que expresan más de una (ver nota a pie anterior). Por otro lado, y con el fin de seleccionar un modelo parsimonioso que ajuste correctamente, se ha utilizado un método de inclusión de variables condicional hacia adelante (Cea D'Ancona 2002). De todos los resultados proporcionados en este texto serán presentados los coeficientes de regresión, el error estándar y la razón de razones, siguiendo la recomendación de Nicol y Pexman (1999). El análisis de la tabla 3 desvela que únicamente la edad y la utilización de tarjetas afectan al número de no respuestas conseguidas en las preguntas entre cuatro y seis categorías de respuesta, mostrando la variable "utilización de tarjetas" mayor influencia que la edad. A medida que aumenta la edad desciende el número de no respuestas, presentando la otra variable (empleo de tarjetas) una influencia notablemente superior.

tados) responden todas, 378 dejan sin responder una pregunta, 8 no responden dos preguntas y 1 deja una pregunta sin responder. 
Tabla 3.

Efecto de las variables sociodemográficas en la no respuesta parcial

\begin{tabular}{|c|c|c|c|c|c|c|}
\hline & \multicolumn{6}{|c|}{ N. ${ }^{\circ}$ de categorías ... } \\
\hline & \multicolumn{3}{|c|}{ Cuatro, cinco y seis } & \multicolumn{3}{|c|}{ Entre ocho y once } \\
\hline & \multicolumn{3}{|c|}{ No sabe } & \multicolumn{3}{|c|}{ No sabe } \\
\hline & \multicolumn{3}{|c|}{$\begin{array}{c}1=\text { Respuesta } \\
0=\text { No respuestas (no sabe) }\end{array}$} & \multicolumn{3}{|c|}{$\begin{array}{c}1=\text { Respuesta } \\
0=\text { No respuestas (no sabe) }\end{array}$} \\
\hline & Coeficientes & $\begin{array}{c}\text { Error } \\
\text { estándar }\end{array}$ & $\begin{array}{l}\text { Odds } \\
\text { ratio }\end{array}$ & Coeficientes & $\begin{array}{c}\text { Error } \\
\text { estándar }\end{array}$ & Odds ratio \\
\hline Uso de tarjetas ( 0 = leídas) & $0,877^{* * *}$ & 0,253 & 2,404 & $0,724^{* \star *}$ & 0,139 & 2,062 \\
\hline Sexo (0 = mujer) &,-- &,-- &,-- & $0,375^{\star \star *}$ & 0,140 & 1,456 \\
\hline Edad & $-0,029^{* * *}$ & 0,008 & 0,971 & $-0,014^{\star * *}$ & 0,005 & 0,986 \\
\hline \multicolumn{7}{|c|}{ Nivel educativo (referencia: superiores) } \\
\hline Sin estudios &,-- &,-- &,-- & $-0,814^{\star * *}$ & 0,312 & 0,443 \\
\hline Primarios &,-- &,-- &,-- & $-0,563^{* *}$ & 0,331 & 0,570 \\
\hline Secundarios y FP &,-- &,-- &,-- & $-0,120$ & 0,361 & 0,886 \\
\hline \multicolumn{7}{|c|}{ Relación con la actividad (referencia: trabajo doméstico no remunerado) } \\
\hline Trabaja &,-- &,-- &,-- &,-- &,-- &,-- \\
\hline Parado &,-- &,-- &,-- &,-- &,-- &,-- \\
\hline Jubilado &,-- &,-- &,-- &,-- &,-- &,-- \\
\hline Estudiante &,-- &,-- &,-- &,-- &,-- &,-- \\
\hline Constante & 3,990 & 0,543 & & 1,780 & 0,395 & \\
\hline$-2 \log$ de Verosimilitud & \multicolumn{2}{|c|}{539,486} & & \multicolumn{2}{|c|}{1245,225} & \\
\hline Pseudo R² (Nagelkerke) & \multicolumn{2}{|c|}{0,067} & & \multicolumn{2}{|c|}{0,104} & \\
\hline$\%$ casos correctos & \multicolumn{2}{|c|}{$92,5 \%$} & & \multicolumn{2}{|c|}{$68,9 \%$} & \\
\hline Número de casos & \multicolumn{2}{|l|}{1805} & & \multicolumn{2}{|l|}{1805} & \\
\hline
\end{tabular}

Notas:

Los coeficientes mostrados son el resultado de una regresión logística "por pasos hacia adelante" considerando como términos independientes sexo, edad, nivel de estudios, relación con la actividad y uso de tarjetas. Únicamente se presentan los términos significativos.

${ }^{*}$ (*) Significación al 0,10.

$\left.{ }^{* *}\right)$ Significación al 0,05.

$\left.{ }^{* * \star}\right)$ Significación al 0,01.

"-,-" Indica relación no significativa

Fuente: Elaboración propia con datos del CIS 2011, Estudio n. ${ }^{0} 2864$. 


\section{Tarjetas con entre ocho y once categorías de respuesta}

En el tercer grupo se han clasificado preguntas con ocho y once categorías de respuesta, que suponen más complicación que las anteriores por la mayor de información que debe considerar el entrevistado. La cantidad de información, considerada por el número de palabras de cada categoría, oscila entre las 47 palabras (319 caracteres) de las preguntas 18 y 21 ( 11 categorías) hasta las 66 palabras (375 caracteres) de la pregunta 12 (10 categorías). En una posición intermedia se sitúa la pregunta 11, con nueve categorías y 58 palabras, y la pregunta 28 que - pese a contar con tres categorías ${ }^{10}$ son "largas" al tener 52 palabras.

\section{Tabla 4.}

Media de no respuestas de las preguntas entre ocho y doce categorías (entre 47 y 66 palabras, 319-375 caracteres)

Preguntas utilizadas:

Preg. 11: razón por la que dejó de hacer trabajo voluntario (tarjeta D, 9 categorías largas, 58 palabras, 355 caracteres).

Preg. 12: razones por las que nunca ha realizado trabajo voluntario (tarjeta E, 10 categorías largas, 66 palabras, 375 caracteres).

Preg.18: motivos por los que usted realizaba labores de voluntariado (tarjeta G, 11 categorías largas, 47 palabras, 319 caracteres).

Preg. 21: motivos por el que hay personas que realizan labores de voluntariado (tarjeta G, 11 categorías largas, 47 palabras, 319 caracteres).

Preg. 28: acuerdo con la necesidad de la reforma del sistema de pensiones (tarjeta 0, 3 categorías largas, 52 palabras, 304 caracteres).

\begin{tabular}{|c|c|c|c|c|c|}
\hline & $\begin{array}{c}\text { Usa } \\
\text { tarjetas }\end{array}$ & $\begin{array}{c}\text { Tarjetas } \\
\text { leídas }\end{array}$ & t-valor & $p$ & $\begin{array}{c}\text { Igualdad } \\
\text { varianzas } \\
\text { (Levene) }\end{array}$ \\
\hline \multicolumn{6}{|c|}{ No responde } \\
\hline Número & 0,1501 & 0,1670 & 0,641 & 0,522 & $\mathrm{Si}$ \\
\hline Porcentaje & $1,8766 \%$ & $2,0872 \%$ & & & \\
\hline \multicolumn{6}{|l|}{ No sabe } \\
\hline Número & 0,3170 & 0,5659 & 5,211 & 0,000 & No \\
\hline Porcentaje & $3,9629 \%$ & $7,0733 \%$ & & & \\
\hline
\end{tabular}

Fuente: Elaboración propia con datos del CIS 2011, Estudio n. ${ }^{0} 2864$.

\footnotetext{
${ }^{10}$ Esta pregunta pudiera haberse clasificado en el grupo primero de tres opciones de respuesta pero la gran cantidad de información que presenta hace que su proceso de respuesta sea más parecido a las preguntas "largas", a las preguntas que incluyen mucha información. Por este motivo se ha decidido clasificarla dentro de este grupo.
} 
La tabla 4 desvela un porcentaje de preguntas no respondidas rondando el $2 \%$, muy similar en ambos grupos. El número de elecciones de la opción "no sabe" es 0,32 en los que usan tarjetas y aumenta hasta el 0,57 entre los que reciben esta información de forma oral, que supone unas tasas de no respuesta del $3,96 \%$ y del $7 \%$ respectivamente. El análisis de regresión (parte derecha de la tabla 3 ) revela la influencia del nivel de estudios, uso de tarjetas, sexo y edad. Las personas sin estudios y con estudios primarios dejan un gran número de preguntas sin responder, cantidad que desciende a medida que aumenta el nivel de estudios. La segunda variable con influencia es uso de tarjetas, seguida del sexo, que indica una mejor cumplimentación del cuestionario por parte de los hombres. La edad, de nuevo, presenta una relación negativa que indica que los jóvenes dejan menos preguntas sin responder que los mayores. De nuevo se observa la gran influencia del empleo de tarjetas, la segunda variable que más influye en el número de elecciones "no sabe".

\section{Tarjetas con doce categorías de respuesta}

Se trata de las preguntas más complicadas del cuestionario por la gran cantidad de información suministrada en la pregunta, alrededor de 100 palabras. El análisis de la tabla 5 desvela diferencias significativas tanto en el número de preguntas no respondidas como en la tasa de "no sabe", teniendo siempre valores más bajos los entrevistados que han respondido con la ayuda de tarjetas. El análisis del efecto de las variables sociodemográficas desvela que el número de preguntas no respondidas está influenciada por el nivel de estudios y la utilización de tarjetas (parte izquierda de la tabla 6).

Tabla 5.

Media de no respuestas de las preguntas con doce categorías

(alrededor de 100 palabras)

Preguntas utilizadas:

Preg. 8: realización de labores de voluntariado en diversas áreas (tarjeta B, 12 categorías largas, 97 palabras, 761 caracteres).

Preg. 15: áreas en las que prestaría trabajo voluntario (tarjeta B, 12 categorías largas, 97 palabras, 761 caracteres).

\begin{tabular}{|c|c|c|c|c|c|}
\hline & $\begin{array}{c}\text { Usa } \\
\text { tarjetas }\end{array}$ & $\begin{array}{l}\text { Tarjetas } \\
\text { leídas }\end{array}$ & t-valor & $p$ & $\begin{array}{c}\text { Igualdad } \\
\text { varianzas } \\
\text { (Levene) }\end{array}$ \\
\hline \multicolumn{6}{|l|}{ No responde } \\
\hline Número & 0,6834 & 0,9091 & 4,363 & 0,000 & Sí \\
\hline Porcentaje & $4,8814 \%$ & $6,4935 \%$ & & & \\
\hline \multicolumn{6}{|l|}{ No sabe } \\
\hline Número & 0,0724 & 0,2208 & 3,693 & 0,000 & No \\
\hline Porcentaje & $0,5113 \%$ & $1,5770 \%$ & & & \\
\hline
\end{tabular}

Fuente: Elaboración propia con datos del CIS 2011, Estudio n. ${ }^{0} 2864$. 


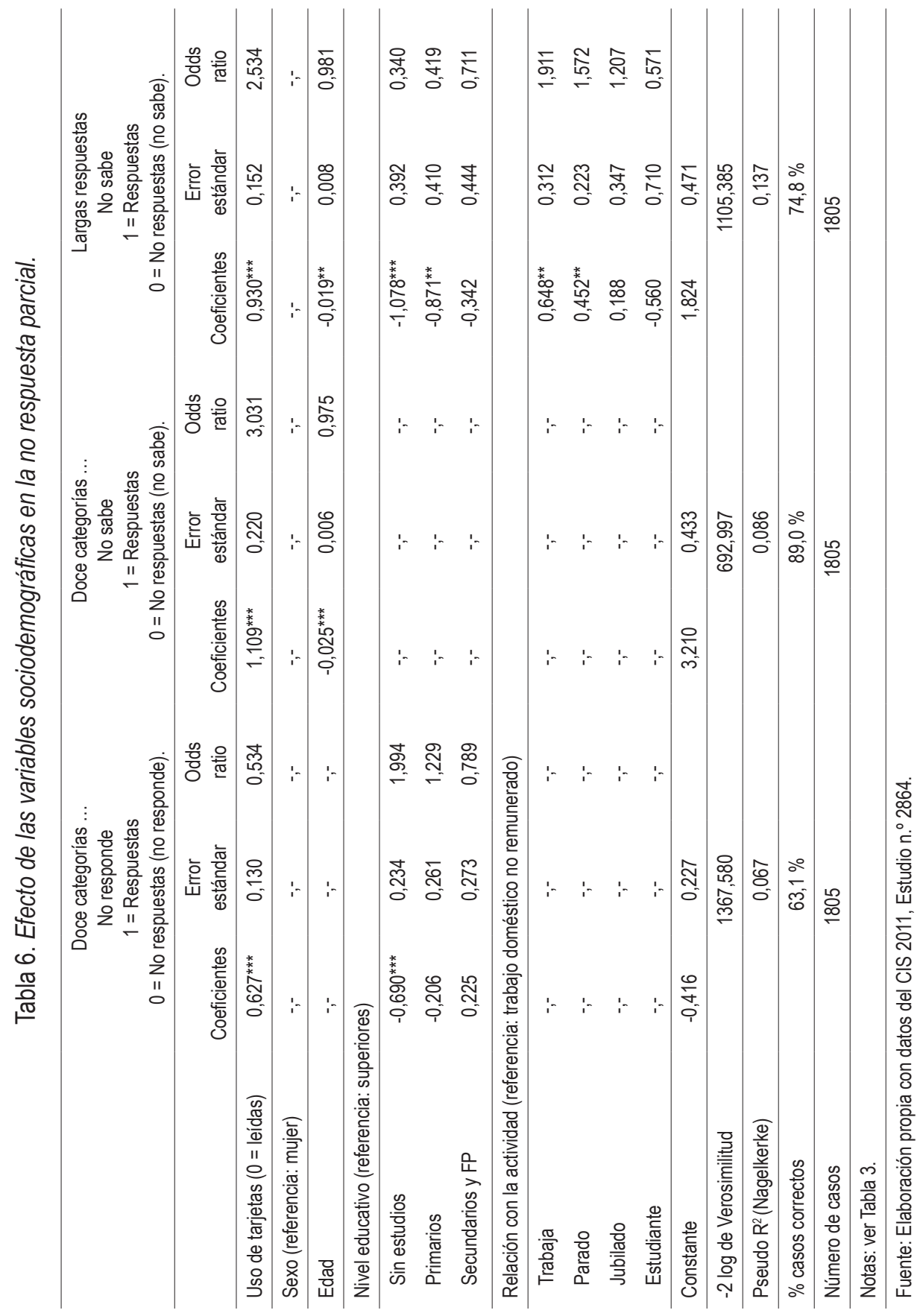


El número de preguntas no respondidas desciende en el grupo de mayores estudios y entre los que usan tarjetas.

El número de respuestas "no sabe" está relacionado con la edad y el uso de tarjetas, mostrando la pauta señalada hasta el momento: los mayores son más reacios a reconocer que no saben, y el número de no respuestas desciende en las personas que utilizan tarjetas.

Recapitulando lo señalado hasta ahora, en todos los casos los entrevistados con tarjeta proporcionan más información que el resto, si bien esta diferencia no es significativa en el número de "no sabe" en las preguntas con tres categorías de respuesta (detectándose en el resto de preguntas). Las no respuestas únicamente presentan diferencias significativas en las preguntas con más de 12 categorías.

\section{LARGAS RESPUESTAS EN PREGUNTAS CON DOS CATEGORÍAS}

El segundo criterio utilizado para la clasificación de las preguntas considera no tanto el número de categorías sino la longitud de la respuesta en preguntas dicotómicas. Se trata de la pregunta 23 que presenta al entrevistado pares de frases para que este seleccione aquella con la que está más de acuerdo. Son frases largas, entre 19 y 31 palabras, y el entrevistado debe expresar su preferencia una vez que estas han sido leídas. La tabla 7 muestra que no hay diferencias significativas en el número de no respuestas, caso distinto de lo que ocurre en la elección de "no sabe". El porcentaje de no sabe en los entrevistados que no utilizaron las tarjetas casi triplica al otro grupo, siendo la mayor diferencia de todas las localizadas hasta ahora. De hecho, siguiendo a Bourque y Clark (1992), que más del $12 \%$ no haya respondido la pregunta plantea serios problemas a la hora de generalizar estos resultados.

El análisis de la influencia de las variables sociodemográficas en la no respuesta desvela la influencia de todas las variables consideradas, a excepción del sexo (parte derecha de la tabla 6). La relación negativa de la edad y los bajos niveles de estudios implica que los mayores y las personas con bajos niveles de estudios proporcionan un elevado número de respuestas "no sabe". La relación con la actividad muestra el escaso número de "no sabe" de los laboralmente activos (ocupados y parados) y la mala cumplimentación por parte de los estudiantes y los dedicados a tareas domésticas no remuneradas. Al igual que ha venido sucediendo, los que utilizan tarjetas proporcionan menos "no sabe" que el resto, siendo la variable que presenta más influencia.

\section{TARJETAS CON ESCALA VISUAL}

El último de los criterios considerados en el cuadro 1 hace referencia a una tarjeta similar a una escala "visual", una "regla" en la cual los entrevistados se posicionan considerando las dimensiones izquierda y derecha. Se plantea como hipótesis que 
Tabla 7.

Número de no respuestas de las preguntas incluidas en el segundo criterio. Largas respuestas en dos categorías

Pregunta utilizada:

Preg. 23, Preg. 23, tarjetas I, J, K, I, M con dos frases cada una:

a) Valoración del papel de la formación en la labor del voluntariado (31 palabras, 200 caracteres);

b) Papel del voluntariado en relación a la cobertura de los servicios públicos (32 palabras, 208 caracteres);

c) Labor resolutiva del voluntariado (22 palabras, 155 caracteres);

d) Papel de la organización en la labor del voluntariado (39 palabras, 252 caracteres) y;

e) Reconocimiento social de la labor del voluntariado (19 palabras, 134 caracteres).

\begin{tabular}{|c|c|c|c|c|c|}
\hline & $\begin{array}{c}\text { Usa } \\
\text { tarjetas }\end{array}$ & $\begin{array}{l}\text { Tarjetas } \\
\text { leídas }\end{array}$ & t-valor & $p$ & $\begin{array}{c}\text { Igualdad } \\
\text { varianzas } \\
\text { (Levene) }\end{array}$ \\
\hline \multicolumn{6}{|l|}{ No responde } \\
\hline Número & 0,0214 & 0,0353 & 0,867 & 0,386 & $\mathrm{Si}$ \\
\hline Porcentaje & $0,4270 \%$ & $0,7050 \%$ & & & \\
\hline \multicolumn{6}{|l|}{ No sabe } \\
\hline Número & 0,2190 & 0,6271 & 7,377 & 0,000 & No \\
\hline Porcentaje & $4,3803 \%$ & $12,5417 \%$ & & & \\
\hline
\end{tabular}

Fuente: Elaboración propia con datos del CIS 2011, Estudio n. ${ }^{\circ} 2864$.

la observación o no de la tarjeta generará importantes cambios en la respuesta, pudiendo implicar la elección de respuestas más extremas, más centradas o más fáciles. Diversas investigaciones han demostrado que las preguntas (de este tipo) que utilizan estímulos visuales (tarjetas) reciben una mayor elección en las categorías centrales (Dillman et al. 2001; Heerwegh y Loosveldt 2008), mientras que las administradas de forma oral proporcionan respuestas más extremas (Tarnai y Dillman 1992).

En la tabla 8 se observa el mayor número de no respuestas y de respuestas "no sabe" por parte en los entrevistados que no han leído las tarjetas, diferencia que prácticamente se duplica en el caso de las respuestas "no sabe". En línea con lo detectado en otros contextos, los que utilizan tarjetas proporcionan unas respuestas más centradas, menos extremas. El análisis de la influencia de las variables sociodemográficas (tabla 9) muestra que el número de no respuestas únicamente está influido por la utilización o no de tarjetas, mientras que las respuestas "no sabe" presentan relación con el sexo, uso de tarjetas y nivel educativo. La interpretación de los coeficientes revela que se mantienen los hallazgos anteriores del bajo número de no respuestas por parte de los entrevistados con altos estudios, los hombres y los que utilizan tarjetas. En este caso el sexo y el nivel educativo muestran una influencia superior al uso de tarjetas. 
Tabla 8.

Número de no respuestas de las preguntas incluidas en el tercer criterio. Tarjetas con escala visual.

Preguntas utilizadas:

Preg. 26: Escala de valoración de la reforma del sistema de pensiones (tarjeta N, escala 0-10, con dos palabras a cada lado).

Preg. 30: Escala de confianza en la gente (tarjeta P, escala 0-10, con dos frases a cada lado).

Preg. 31: Escala ideología (tarjeta Escala, escala 1-10, con una palabra a cada lado).

\begin{tabular}{|c|c|c|c|c|c|}
\hline & $\begin{array}{c}\text { Usa } \\
\text { tarjetas }\end{array}$ & $\begin{array}{l}\text { Tarjetas } \\
\text { leídas }\end{array}$ & t-valor & $p$ & $\begin{array}{c}\text { Igualdad } \\
\text { varianzas } \\
\text { (Levene) }\end{array}$ \\
\hline \multicolumn{6}{|l|}{ No responde } \\
\hline Número & 0,1149 & 0,1929 & 3,435 & 0,001 & No \\
\hline Porcentaje & $3,9311 \%$ & $6,4317 \%$ & & & \\
\hline \multicolumn{6}{|l|}{ No sabe } \\
\hline Número & 0,1814 & 0,3618 & 5,251 & 0,000 & No \\
\hline Porcentaje & $14,5277 \%$ & $22,2845 \%$ & & & \\
\hline
\end{tabular}

Fuente: Elaboración propia con datos del CIS 2011, Estudio n. 2864. 
Tabla 9.

Efecto de las variables sociodemográficas en la no respuesta parcial

\begin{tabular}{|c|c|c|c|c|c|c|}
\hline & \multicolumn{6}{|c|}{ Escala visual } \\
\hline & \multicolumn{3}{|c|}{ No responde } & \multicolumn{3}{|c|}{ No sabe } \\
\hline & \multicolumn{3}{|c|}{1 = Respuestas } & \multicolumn{3}{|c|}{$1=$ Respuestas } \\
\hline & \multicolumn{3}{|c|}{$0=$ No respuestas (no responde) } & \multicolumn{3}{|c|}{$0=$ No respuestas (no sabe) } \\
\hline & Coeficientes & $\begin{array}{c}\text { Error } \\
\text { estándar }\end{array}$ & Odds ratio & Coeficientes & $\begin{array}{c}\text { Error } \\
\text { estandar }\end{array}$ & Odds ratio \\
\hline Uso de tarjetas ( 0 = leídas) & $0,546^{\star * *}$ & 0,178 & 1,727 & $0,689^{* * *}$ & 0,156 & 1,992 \\
\hline Sexo (0 = mujer) &,-- &,-- &,-- & $0,722^{* * *}$ & 0,161 & 2,059 \\
\hline Edad &,-- &,-- &,-- &,-- &,-- &,-- \\
\hline \multicolumn{7}{|c|}{ Nivel educativo (referencia: superiores) } \\
\hline Sin estudios &,-- &,-- &,-- & $-1,340^{\star \star *}$ & 0,405 & 0,262 \\
\hline Primarios &,-- &,-- &,-- & $-1,088^{* *}$ & 0,432 & 0,337 \\
\hline Secundarios y FP &,-- &,-- &,-- & $-0,472$ & 0,466 & 0,624 \\
\hline \multicolumn{7}{|c|}{ Relación con la actividad (referencia: trabajo doméstico no remunerado) } \\
\hline Trabaja &,-- &,-- &,-- &,-- &,-- &,-- \\
\hline Parado &,-- &,-- &,-- &,-- &,-- &,-- \\
\hline Jubilado &,-- &,-- &,-- &,-- &,-- &,-- \\
\hline Estudiante &,-- &,-- &,-- &,-- &,-- &,-- \\
\hline Constante & 1,531 & 0,113 & & 1,797 & 0,403 & \\
\hline-2 log de Verosimilitud & 874,858 & 1051,731 & & & & \\
\hline Pseudo R² (Nagelkerke) & 0,016 & 0,094 & & & & \\
\hline$\%$ casos correctos & $85,5 \%$ & $78,3 \%$ & & & & \\
\hline Número de casos & 1805 & 1805 & & & & \\
\hline \multicolumn{7}{|c|}{$\begin{array}{l}\text { Notas: } \\
\text { Los coeficientes mostrados son el resultado de una regresión logística "por pasos hacia adelante" considerando } \\
\text { como términos independientes sexo, edad, nivel de estudios, relación con la actividad y uso de tarjetas. Única- } \\
\text { mente se presentan los términos significativos. } \\
\left({ }^{*}\right) \text { Significación al } 0,10 . \\
\left({ }^{* *}\right) \text { Significación al } 0,05 \text {. } \\
\left(^{* * *} \text { Significación al } 0,01 \text {. }\right. \\
\text { "-,-" Indica relación no significativa. }\end{array}$} \\
\hline
\end{tabular}

Fuente: Elaboración propia con datos del CIS 2011, Estudio n. ${ }^{\circ} 2864$. 


\section{CONCLUSIONES}

Comenzábamos este trabajo pensando en un entrevistador que, en la puerta de un domicilio (la mayor parte de las veces), tiene que "administrar" 23 tarjetas mientras lee las preguntas del cuestionario y anota las respuestas del entrevistado. Como no todas las preguntas se responden con la ayuda de estos "recursos visuales" el entrevistador realiza la pregunta - con la tarjeta - cuando el cuestionario así lo indica, y la guarda si la siguiente pregunta no precisa de su utilización, ya que su presencia podría confundir al encuestado. La experiencia nos advierte de que son demasiadas tareas para un entrevistador que, además, debe mantener el contacto con el encuestado, convencerle para que acepte responder a la entrevista, recoger con precisión su respuesta -muy complicado cuando las preguntas son abiertas-, etc. (entre otros, De Leeuw 2008; Schaeffer, Dykema y Maynard 2010).

Trabajos realizados por otros investigadores han advertido que la forma habitual en la que son administradas las tarjetas difiere de lo planificado y deseado por el investigadordiseñador del cuestionario (Díaz de Rada y Núñez Villuendas 2008). En el caso concreto de este estudio, las respuestas de los entrevistadores a la pregunta D50 desvela que algo más de la mitad de los encuestados (un 53,7 \%) ha utilizado las tarjetas como indicó el diseñador del cuestionario, mientras que casi uno de cada cuatro (exactamente un $23,7 \%$ ) ha utilizado algunas - sin indicar cuáles-y el $22 \%$ no utilizaron ninguna, por lo que el entrevistador procedió con una lectura de la información incluida en las tarjetas. Considerábamos que esta forma de proceder provocaría un aumento del tiempo necesario para la cumplimentación del cuestionario, algo que no sucede porque la diferencia en el tiempo medio de respuesta entre el primer grupo (lee tarjetas) y el tercero (escucha texto tarjetas, lectura por el entrevistador) apenas difiere en treinta segundos (18,13 minutos frente a 18,43 respectivamente). 0 los entrevistadores del CIS leen (en voz alta) con la misma rapidez que los encuestados (leen para sí, en bajo), o los tiempos de cumplimentación del cuestionario no se recogen con suficiente precisión. Mucho nos tememos, observando el espacio dedicado en el cuestionario para tal fin, que se trata de la segunda situación (Lynn 1998: 19).

A lo largo del trabajo se ha mostrado que el número de respuestas "no sabe" es mayor entre los que "escuchan" las tarjetas, diferencia que aumenta a medida que aumenta el número de información en las opciones de respuesta. Los rasgos sociodemográficos de los entrevistados influyen menos que la utilización/no utilización de tarjetas cuando se trata de preguntas hasta once categorías (tabla 3); aumentando la influencia del nivel educativo y la relación con la actividad en las preguntas de doce categorías. En las no respuestas las diferencias entre los grupos son muy inferiores, afectando únicamente en las preguntas con doce categorías de respuesta.

Consideramos que en las escalas visuales impera otra lógica, una lógica de representación espacial, puesto que las diferencias en el número de palabras es muy baja ${ }^{11}$.

\footnotetext{
${ }^{11}$ La pregunta 26 cuenta con cuatro palabras (muy negativamente / muy positivamente), la pregunta 38
} 
En estas tres preguntas existen importantes diferencias, tanto en el número de "no sabe" como en las no respuestas, donde los entrevistados que no usan las tarjetas prácticamente doblan a los entrevistados con tarjetas.

Terminaremos el trabajo considerando las valoraciones que realizan los encuestadores de la dificultad que - desde su punto de vista - han encontrado los encuestados en la comprensión de determinadas preguntas. Se utilizarán para ello las respuestas a la pregunta 48 que plantea a los encuestadores si "ha habido alguna pregunta concreta que la persona entrevistada tuviera dificultades en comprender o que tuviera que ser explicada", pudiendo proporcionar hasta cinco respuestas ${ }^{12}$.

Los entrevistadores localizaron únicamente 105 personas con dificultades en la comprensión de algunas preguntas (un 4,3\%), que se reducen a 58 (2,4\%) cuando se consideran los dos colectivos empleados en esta investigación: los que utilizaron todas las tarjetas, y los que ninguna. Aun al tratarse de un bajo número - con escasa representatividad - hay más personas con dificultades en el grupo que ha "escuchado" las tarjetas que entre los que las han utilizado. Analizando con detalle las respuestas de las personas que han respondido sin leer las tarjetas destacan las preguntas de escala - definidas en el cuadro 1 como "tarjetas visuales"- que han generado problemas para el $83 \%$ de los entrevistados: la escala de valoración de la reforma de pensiones (pregunta 26) presenta problemas para el $36 \%$, la escala de confianza en la gente (pregunta 30) para un $29 \%$, y la ideología (pregunta 31) para el $18 \%$. Del resto de preguntas señalar que un tercio de los entrevistados sin tarjeta encontraron difícil la pregunta 28 (entre 8 y 11 categorías, algo menos del 20\% la pregunta y 31 (escala ideológica) y 14\% las preguntas 8 y 15 (preguntas con la misma respuesta, doce categorías y alrededor de 100 palabras $)^{13}$. En la primera de estas preguntas el porcentaje de entrevistados con dificultades es similar en ambos grupos, hayan escuchado o leído las tarjetas. Resulta sorprendente que un $16 \%$ de los entrevistados que han leído las tarjetas hayan tenido dificultades en la pregunta 23 (largas respuestas en dos categorías), porcentaje que se reduce al $4 \%$ en el grupo que escucha esta información. Recomendamos suma prudencia en la interpretación de estos resultados porque se circunscriben únicamente a 105 personas de las 2461 entrevistadas.

Como conclusión final, el escaso número de preguntas sin responder (baja tasa de no respuesta parcial) entre los que responden con tarjetas permite afirmar que la utilización de estas ayudas visuales supone importantes mejoras en el proceso de respuesta del cuestionario; aún cuando se empleen cuestionarios con muchas tarjetas y estas

algunas más (15: nunca se es lo bastante prudente / se puede confiar en la mayoría de la gente) y dos la pregunta 31 (izquierda / derecha).

${ }^{12}$ Texto de la pregunta 48 (respondida por los entrevistadores): “¿Ha habido alguna pregunta concreta que la persona entrevistada tuviera dificultades en comprender o que tuviera que ser explicada?" (máximo cinco respuestas).

${ }^{13}$ Las respuestas superan el 100 porque se recogen hasta cinco respuestas. 
incluyan gran cantidad de información. Debe tenerse en cuenta que se ha utilizado un caso "extremo" para llevar a cabo esta comprobación, caso extremo definido por el elevado número de tarjetas empleadas (23 tarjetas) y la longitud de algunas de estas (más de 100 palabras). Si en este caso "extremo" la utilización de tarjetas supone mejoras en el proceso de respuesta del cuestionario, sin duda estas funcionarán mejor en cuestionarios que empleen menos tarjetas de respuestas o tarjetas menos complejas que las empleadas aquí.

Antes de finalizar conviene tener presente una importante limitación de este trabajo. Debe tenerse en cuenta que los entrevistados no han sido asignados aleatoriamente en dos grupos diferentes, sino que se ha utilizado la declaración del encuestador sobre si el entrevistado había utilizado las tarjetas o no. Pese a que los datos han sido ponderados utilizando tres variables sociodemográficas (sexo, edad, nivel educativo y relación con la actividad, ver Anexo 2), pueden existir diferencias en las variables propias de la investigación. De hecho, los que utilizan las tarjetas presentan una mayor tasa de trabajo voluntario que el resto. Pudiera ser también que aquellos que emplean las tarjetas estuvieran más interesados por la encuesta 0 , al menos, más complacientes con la tarea demandada por el entrevistador. Además, pueden existir encuestadores más exigentes a la hora de aplicar el cuestionario, que traten de seguir el protocolo fijado insistiendo a los entrevistados a utilizar las tarjetas, mientras que otros pueden considerarlas como "accesorias" y no insistir tanto en su correcta lectura. En relación a este último aspecto se ha buscado conocer la influencia del entrevistador comparando el uso de tarjetas por cada entrevistador con la distribución total, esto es, la distribución "intra-entrevistador" con la distribución "inter-entrevistador". Esta operación permite apreciar la existencia de una gran variabilidad dentro de cada entrevistador, lo que "destierra" la idea de la influencia del entrevistador.

Todos estos son factores que limitan la generalidad de los hallazgos aquí localizados, y animan a realizar más investigación sobre estos aspectos. Pero es preciso indicar que se trata de un problema que está presente en un gran número de investigaciones internacionales publicadas en las revistas de más impacto. Así, por ejemplo, son numerosos los estudios sobre no respuesta (total) en las encuestas donde el tema de la encuesta interactúa con la mayor o menor propensión a responder; y el estudio se presenta como un "caso" de los factores que influyen en la propensión a responder. Véanse, por ejemplo, el clásico estudio de Catania y otros (1996) sobre la influencia del sexo del entrevistado en la encuesta americana de hábitos sexuales, así como los trabajos de Abrahan et al. (2006), Johnson et al. (2006), y Keeter et al. (2006) ${ }^{14}$.

\footnotetext{
${ }^{14}$ 2006. Public Opinion Quarterly 5 (número monográfico sobre no respuesta).
} 


\section{REFERENCIAS BibLIOGRÁFICAS}

Abraham, K. et al. 2006. "Nonresponse in the American Time Use survey: Who is missing from the data and how much does it matter?". Public Opinion Quarterly 70:676-703.

Azofra, M. J. 1999. Cuestionarios. Madrid: CIS (Colección Cuadernos Metodológicos 26).

Berdie, D. y John F. Anderson. 1974. Questionnaires: design and use. Metuchen, Nj: Scarerow.

Bishop, G. F. y A. Smith 2001. "Response-order effects and the early Gallup split-ballots". Public Opinion Quarterly 65:479-505.

Bishop, G. F., A. Oldendick y A. Tuchfarber. 1983. "Effects of filter questions in Public Opinion surveys". Public Opinion Quarterly 47:528-546.

Bourque, L. B. y V. A. Clark. 1992. Processing Data: The Survey Example, Sage University Paper Series on Quantitative Applications in the Social Sciences 07-085. Newbury Park, CA: SAGE.

Catania, J. A. et al. 1996. "Effects of Interviewer Gender, Interviewer Choice, and Item Wording of Responses to Questions Concerning Sexual Behavior". Public Opinion Quarterly 60:345-375.

Cea D’Ancona, M. Á. 2002. Análisis Multivariable. Teoría y práctica en la investigación social. Madrid: Síntesis.

Centro de Investigaciones Sociológicas 2011. Barómetro de marzo. Madrid: CIS, estudio número 2864.

Centro de Investigaciones Sociológicas. 2011. Normas generales para la correcta aplicación del cuestionario. Madrid: $\mathrm{CIS}$ (documento no publicado).

Chang, L. y J. A. Krosnick. 2010. "Comparing oral interviewing with self-administered computerized questionnaires: an experiment". Public Opinion Quarterly 74:154-167.

Converse, J. M. 1964. "Predicting no opinion in the polls". Public Opinion Quarterly 40:515-530.

Converse, J. M y S. Presser. 1986. "Survey Questions: handcrafting the standardized questionnaire". Sage University Paper series on Quantitative Applications in the Social Sciences 63. Beverly Hills y Londres: Sage.

De Leeuw, E. 2008. "Choosing the method of data collection". Pp. 113-135 en International Handbook of Survey Methodology, editado por Edith D. de Leeuw, Joop J. Hox y Don A. Dillman. Nueva York: Lawrence Erlbaum Associates y Asociación Europea de Metodología.

Díaz de Rada, V. 2001. Diseño y elaboración de cuestionarios para la investigación comercial. Madrid: ESIC.

Díaz de Rada, V. y A. Núñez Villuendas. 2008. Estudio de las incidencias en la investigación mediante encuesta. Madrid: Centro de Investigaciones Sociológicas.

Dillman, Don A., G. Phelps, R. Tortora, K. Swift, J. Kohrell y J. Berck. 2001. "Response rate and measurement differences in mixed mode surveys using mail, telephone, interactive voice response and the Internet", Social and Economic Sciences Research Center, Washington State University, Department of Sociology. Washington http://survey.sesrc.wsu.edu/dillman/papers.htm.

Dillman, D. A., Jolene D. Smyth y M. C. Leah 2009. Internet, Mail and Mixed-Mode Surveys: The Tailored Design Method. Nueva York: Wiley. 
Gilljam, M. y D. Granberg. 1993. "Should we take don't know for an answer?". Public Opinion Quarterly 57:348-357.

Groves, R. M. y R. L. Kahn. 1979. Surveys by telephone: a national comparison with personal interviews. Orlando, FL.: Academic Press.

Heerwegh, D. 2009. "Mode differences between face-to-face and web surveys: An experimental investigation of data quality and social desirability effects". International Journal of Public Opinion Research 21:111-121.

Heerwegh, D. y G. Loosveldt. 2008. "Face to face versus web surveying in a high internet coverage population". Public Opinion Quarterly 72:836-846.

Holbrook, A L., M. C. Green y M. C. Krosnick. 2003. "Telephone versus face-to-face interviewing of national probability samples with long questionnaires". Public Opinion Quarterly 67:79-125.

Holbrook, A. L. y J. A. Krosnick. 1999. Response Order effects in telephone interviews. The impact of linguistic structure, and respondent ability, motivation and task difficulty. Paper presented at the annual conference of the American Association for Public Opinion Research, St. Petesburg Beach, FL.

Holbrook, A. L., J. A., Krosnick, D. Moore y R. Tourangeau. 2007. "Response order effects in dichotomous categorical questions presented orally: the impact of question and respondent attributes". Public Opinion Quarterly 71:325-348.

Johnson, T. et al. 2006. "Using Community-Level correlates to evaluate nonresponse effects in a telephone survey". Public Opinion Quarterly 70:704-719.

Keeter, S. et al. 2006. "Gauging the impact of growing nonresponse on estimates from a national RDD telephone survey". Public Opinion Quarterly 70:759-779.

Krosnick, J. A., A. L. Holbrook, M. K. Berent, R. T. Carson, W. M. Hanemann, R. J. Kopp, R. C. Mitchell, S. Presser, P. A. Ruud, V. Kerry Smith, W. R. Moody, M. C. Green y M. Conaway. 2002. "The Impact of 'No Opinion' Response Options on Data Quality: Non-Attitude Reduction or an Invitation to Satisfice?". Public Opinion Quarterly 66:371-403.

Lynn, P. 1998. "Data collection mode effects on responses to attitudinal questions". Journal of Official Statistics 14:1-14.

Nicol, Adelheid A. M. y Penny M. Pexman. 1999. Presenting your findings: A practical guide for creating tables. Washington: American Phychological Association.

Oppenheim, A. N. 1992. Questionnaire design. Interviewing and attitude measurement. Londres: Pinter.

Petersen, T. 2008. "Split Ballots as an experimental approach to public opinion research". Pp. 322-329 en The SAGE Handbook of Public Opinion Research, editado por W. Donsbach y M. W. Traugott. Thousand-Oaks, LA: Sage.

Survey Research Laboratory, University of Illinois. 1994. General Training Manual for Face-to-Face Interviewers. Chicago, Illinois: Universidad de Illinois.

Tarnai, J. y D. A. Dillman. 1992. "Questionnaire context as a source of response differences in mail and telephone survey". Pp. 115-129 en Context effects in social and psychological research, editado por Norbert Schwarz y Seymour Sudman. Nueva York: Springer. 
Schaeffer, N. C., J. Dykema y D. W. Maynard. 2010. "Interviewers and interviewing". Pp. 437-470 en Handbook of Survey Research, editado por Peter V. Marsden y James D. Wright. Bingley UK: Emerald Group Publishing Limited.

Schuman, H. y S. Presser. 1996. Questions and answers in attitudes surveys. Londres: Sage.

VIDAL Díaz de Rada IgúzQuIZa es Licenciado y Doctor en Sociología, Licenciado en Investigación y Técnicas de Mercado y profesor de Sociología en el Departamento de Sociología de la Universidad Pública de Navarra. Es autor de diversos trabajos sobre metodología, sociología del consumo y comportamiento del consumidor; destacando entre sus últimas publicaciones: "Encuestas con encuestador y encuestas autoadministradas por internet. ¿Proporcionan resultados comparables?" (2011), "Ventajas e inconvenientes de la encuesta por internet" (2012) y "La no respuesta en las encuestas presenciales realizadas en España" (2013).

RECIBIDO: 05/12/2012

ACEPTADO: 30/06/2013

Publicado on-line: 13/03/2014 


\section{AnEXos}

Anexo 1.

Variables incluidas en el Barómetro de marzo de 2011 (Estudio CIS n. ${ }^{\circ}$ 2864)

\section{Preguntas fijas todos los meses:}

- Preg. 1: Valoración de la situación económica actual.

- Preg. 2: Valoración de la situación económica con respecto a la situación hace 1 año.

- Preg. 3: Valoración de la situación política actual.

- Preg. 4: Valoración de la situación política con respecto a la situación hace 1 año.

- Preg. 5: Principales problemas del país en opinión de la persona entrevistada.

- Preg. 6: Problemas que más afectan a la persona entrevistada.

Preguntas específicas del Barómetro de marzo, con indicación de las preguntas con tarjetas:

- Preg. 7: Frecuencia con la que ha realizado diversas actividades sin ánimo de lucro.

\section{Tarjeta A, 3 categorías cortas.}

- Preg. 8: Realización de labores de voluntariado en diversas áreas: donar sangre, donar dinero, entregar ropa, cuidado de otras personas son cobrar, actividades en beneficio de la comunidad, y actividades colectivas de interés general. Tarjeta $\mathbf{B}$, 12 categorías largas.

- Preg. 9: Realización de labores de voluntariado en diversas áreas en el último año: defensa de derechos humanos, exclusión social, discapacidad y dependencia, educación y cultura, salud, deporte y ocio, protección de animales, cooperación al desarrollo, protección civil, y voluntariado en cualquier área.

- Preg. 10 y 10a: A quienes han realizado labores de voluntariado antes del último año: frecuencia (Tarjeta C, 4 categorías cortas) y número de horas dedicadas.

- Preg. 11: Razón para dejarlas Tarjeta D, 8 categorías largas.

- A quienes no han realizado nunca labores de voluntariado: - Preg. 1201 y 1202: razón para no realizarlas nunca.

- Preg. 13: Existencia de familiares y amigos que realizan trabajo voluntario.

- Preg. 14: Intención de involucrarse o participar en actividades de trabajo voluntario.

- Preg. 1501 1502: Áreas en las que prestaría trabajo voluntario. Tarjeta B, 12 categorías largas.

- Voluntarios los últimos 12 meses:

-Preg. 16 y 16a: frecuencia y número de horas dedicadas, Tarjeta C, 4 categorías cortas. 
- Preg. 17: asociación/organización en la que ha realizado trabajo voluntario. Tarjeta F, 11 categorías largas.

- Preg. 18: motivos para realizar trabajo voluntario. Tarjeta G, 11 categorías largas.

- Preg. 19: Satisfacción trabajo voluntario, resultados, funcionamiento de organización.

- Preg. 20: Realización de labores de voluntariado por familiares y amigos/as antes de que usted hiciera trabajo voluntario.

- Preg. 21: Motivos por el que hay personas que realizan labores de voluntariado. Tarjeta G, 11 categorías largas.

- Preg. 22: Motivos por el que hay personas que NO realizan labores de voluntariado. Tarjeta $\mathrm{H}, 6$ categorías medianas.

- Preg. 23: Valoración del papel de la formación en la labor del voluntariado, del papel del voluntariado en relación a la cobertura de los servicios públicos, de la labor resolutiva del voluntariado, del papel de la organización en la labor del voluntariado y del reconocimiento social de la labor del voluntariado. Tarjeta I, J, $\mathrm{K}, \mathrm{I}, \mathrm{M}$, dos frases largas.

- Preg. 24 y 24a: Beneficiario/a (entrevistado/a o familiares o amigos/as) de la labor de los/as voluntarios/as.

- Preg. 25: Conocimiento del Año Europeo del Voluntariado (2011).

- Preg. 26: Escala de valoración de la reforma del sistema de pensiones. Tarjeta N, escala 1-10, con dos palabras a cada lado.

- Preg. 27: Acuerdo con distintas medidas tomadas en la reforma del sistema de pensiones: ampliar la edad de jubilación a los 67 años, calcular la pensión con 25 años de cotización, recepción el total de la pensión si ha cotizado 37 años,...

- Preg. 28: Acuerdo con la necesidad de la reforma del sistema de pensiones. Tarjeta 0 , tres frases largas.

- Preg. 29: Evolución de la opinión sobre el Gobierno, los sindicatos y las organizaciones empresariales tras la reforma del sistema de pensiones.

- Preg. 30: Escala de confianza en la gente. Tarjeta P, escala 1-10, con dos frases a cada lado.

\section{Rasgos sociodemográficos (al final del cuestionario)}

- Sexo.

- Edad alcanzada.

- Máximo nivel de estudios alcanzado.

- Religiosidad del entrevistado.

- Frecuencia de asistencia a oficios religiosos.

- Calificación de la situación económica personal en la actualidad.

- Estado civil.

- Persona que aporta más ingresos al hogar. 
- Situación laboral (relación con la actividad): trabajada, parados, jubilado, estudiante, trabajo doméstico no remunerado.

- Solo trabajadores: Probabilidad de que pierda el trabajo en el próximo año.

- Solo parados: Probabilidad de que encuentre trabajo en el próximo año.

- Relación con la actividad: trabajada, parados, jubilado, estudiante, trabajo doméstico no remunerado.

- Ocupación u oficio.

- Situación profesional: asalariado, empresario, autónomo, ayuda familiar, cooperativista, otros.

- Titularidad (privado/estatal) del lugar de trabajo (Administración Pública, empresa pública, empresa privada, organización sin ánimo de lucro, servicio doméstico y otros).

- Actividad principal de la empresa u organización donde trabaja la persona que aporta más ingresos al hogar.

- Nacionalidad española (de nacimiento o adquirida con posterioridad).

- Manejo del castellano.

- Número de teléfono (para comprobación de la entrevista). 
Anexo 2.

Distribución muestral ponderada considerando el sexo, edad, nivel educativo y relación con la actividad (porcentajes de columna)

Tabla de contingencia SEXO y TARJETAS

\begin{tabular}{l|l|c|c|c}
\hline \multicolumn{5}{|c}{$\%$ dentro de TARJETAS } \\
\hline \multirow{2}{*}{ SEXO } & & \multicolumn{2}{c}{ TARJETAS } & Total \\
& Hombre & $45,2 \%$ & $44,9 \%$ & $45,1 \%$ \\
& Mujer & $54,8 \%$ & $55,1 \%$ & $54,9 \%$ \\
\hline Total & & $100,0 \%$ & $100,0 \%$ & $100,0 \%$ \\
\hline
\end{tabular}

Tabla de contingencia EDAD_CUO y TARJETAS

\begin{tabular}{l|l|c|c|c}
\hline \multicolumn{5}{|c}{$\%$ dentro de TARJETAS } \\
\hline & & \multicolumn{2}{c}{ TARJETAS } & Total \\
\hline EDAD_CUO & $18-24$ años & Ha utilizado todas & Las he leído yo & $4,1 \%$ \\
& $25-34$ años & $4,1 \%$ & $4,1 \%$ & $8,7 \%$ \\
& $35-44$ años & $8,7 \%$ & $8,7 \%$ & $9,6 \%$ \\
& $45-54$ años & $9,6 \%$ & $9,6 \%$ & $12,6 \%$ \\
& $55-64$ años & $12,7 \%$ & $12,4 \%$ & $14,8 \%$ \\
& 65 y más años & $14,8 \%$ & $14,8 \%$ & $50,2 \%$ \\
\hline
\end{tabular}

Tabla de contingencia Estudios del entrevistado y TARJETAS

\begin{tabular}{l|l|c|c|c}
\hline \multicolumn{5}{c}{$\%$ dentro de TARJETAS } \\
\hline & & \multicolumn{2}{c}{ TARJETAS } & Total \\
\hline \multirow{2}{*}{$\begin{array}{l}\text { Estudios del } \\
\text { entrevistado }\end{array}$} & Sin estudios & $58,9 \%$ & $58,9 \%$ & $58,9 \%$ \\
& Primarios & $18,8 \%$ & $18,8 \%$ & $18,8 \%$ \\
& Secundarios y FP & $14,1 \%$ & $14,1 \%$ & $14,1 \%$ \\
& Superiores & $8,2 \%$ & $8,2 \%$ & $8,2 \%$ \\
\hline Total & & $100,0 \%$ & $100,0 \%$ & $100,0 \%$ \\
\hline
\end{tabular}


352 • VIDAL DÍAZ DE RADA IGÚZQUIZA

Tabla de contingencia Relación con la actividad y TARJETAS

\begin{tabular}{|c|c|c|c|c|}
\hline \multicolumn{5}{|c|}{$\%$ dentro de TARJETAS } \\
\hline & & \multicolumn{2}{|c|}{ TARJETAS } & \multirow[b]{2}{*}{ Total } \\
\hline & & Ha utilizado todas & Las he leído yo & \\
\hline \multirow[t]{5}{*}{ RELAC } & Trabaja & $24,8 \%$ & $23,0 \%$ & $23,9 \%$ \\
\hline & $\begin{array}{l}\text { Jubilado/a o pensionista } \\
\text { (anteriormente ha trabajado) }\end{array}$ & $51,3 \%$ & $50,5 \%$ & $50,9 \%$ \\
\hline & Parado/a y ha trabajado antes & $12,0 \%$ & $11,0 \%$ & $11,5 \%$ \\
\hline & Estudiante & $1,5 \%$ & $1,9 \%$ & $1,7 \%$ \\
\hline & $\begin{array}{l}\text { Trabajo doméstico no } \\
\text { remunerado }\end{array}$ & $10,4 \%$ & $13,6 \%$ & $12,0 \%$ \\
\hline Total & & $100,0 \%$ & $100,0 \%$ & $100,0 \%$ \\
\hline
\end{tabular}

